ARTÍ́CULO DE REVISIÓN

\title{
La carga de la diabetes en América Latina y el Caribe: análisis a partir de los resultados del Estudio Global de Carga de Enfermedad del año 2015
}

Emma M. Domínguez Alonso*

Departamento de Metodología de la Investigación, Instituto Nacional de Endocrinología, La Habana, Cuba

\section{RESUMEN}

La diabetes mellitus (DM) constituye uno de los fundamentales problemas de salud en América Latina y el Caribe (AL-C), pues es la cuarta causa de pérdidas de vida saludable (AVISA), llegando a ocupar el primer lugar en varios países de la región.

En este trabajo se integran y analizan algunos resultados para la DM aportados por el Estudio Global de Carga de Enfermedad (2015), conducido por el Instituto de Métricas de Salud y Evaluación. Estos resultados evidencian un panorama desfavorable de la región cuando se comparan los diferentes indicadores analizados con los correspondientes valores globales. Tres cuartas partes de los países de la región tienen AVISA debidas a la DM superiores al promedio global.
ABSTRACT

Diabetes mellitus is one of the most important health problems in Latin America and the Caribbean. It is the fourth cause of healthy life loss, reaching the first place in several countries in the region.

In this paper we integrate and analyze some diabetes results from the Global Burden of Disease Study (2015), conducted by the Institute of Health Metrics and Evaluation. These results show an unfavorable panorama of the region when comparing the different indicators analyzed with the corresponding global values. Three-quarters of the countries in the region have healthy diabetes-related life losses above the global average. The regions that consistently show a greater burden are the Caribbean and central Latin
Correspondencia:

*Emma M. Domínguez Alonso

E-mail: emmadadinfomed.sld.cu
Fecha de recepción: 27-09-2017

Fecha de aceptación: 29-01-2018

Doi:10.24875/ALAD.18000314 
Las regiones que consistentemente muestran una mayor carga son el Caribe y Latinoamérica central. Adicionalmente, se comenta sobre algunos de los factores influyentes en la situación de la DM en la región y se discute sobre cambios de estrategias que resultan esenciales para revertirla.

Palabras clave: Diabetes mellitus. Años de vida saludable perdidos. Años perdidos por mortalidad prematura. Años vividos con discapacidad. América Latina y el Caribe. Inequidades en salud.
America. In addition, some of the influential factors in the diabetes situation in the region are commented, and changes in strategies that are essential to revert it are discussed. (Rev ALAD. 2018;8:81-94)

Corresponding author: Emma M. Domínguez Alonso, emmada@infomed.sld.cu

Key words: Diabetes mellitus. Disability-adjusted live years. Years lived with disability. Years of life loss, premature mortality. Latin America and the Caribbean. Health inequalities. Health care inequities.

\section{INTRODUCCIÓN}

El marcado incremento de las personas con DM durante las últimas décadas ha sido catalogado como epidémico, por lo que esta enfermedad es considerada uno de los fundamentales problemas de salud a afrontar a nivel global. Este comportamiento ha resultado, esencialmente, del incremento de la incidencia asociado al envejecimiento poblacional y a los cambios de estilos de vida propios de la urbanización ${ }^{1,2}$.

Estimados de la Federación Internacional de Diabetes (IDF) para el año 2015 reportaron un $8.8 \%$ de la población adulta mundial (alrededor de 415 millones de personas) viviendo con la enfermedad, tres cuartas partes de ellas residiendo en países de medianos y bajos ingresos. Ese año ocurrieron, aproximadamente, cinco millones de fallecimientos como consecuencia de la DM; la mitad de ellos en personas menores de 60 años 3 .

Las predicciones no auguran un mejor escenario en relación con la afectación por DM. En el año 2015, la IDF predijo un incremento de 227 millones de casos (55\%) para 2040 a nivel global. Estas cifras difieren entre regiones, fluctuando desde el 19 al 141\% en Europa y África, respectivamente ${ }^{3}$.

Todo esto confluye en una elevada carga económica para las familias y los sistemas de salud. Los gastos generados por la DM a nivel global alcanzaron la cifra de 548 miles de millones de dólares en el año 2013 , el 11\% del total de los gastos de salud en adultos, y la cifra prevista para 2035 es de 627 miles de millones de dólares; aunque se observan importantes diferencias entre regiones en este aspecto ${ }^{4}$.

En los países de AL-C la DM también representa una importante carga. Entre todas las regiones de la IDF, América del Norte y el Caribe es la de mayor prevalencia, con un $12.9 \%$ en 2015 y predicciones de alcanzar un $14.7 \%$ para $2040^{3}$. Es, a su vez, la región con mayores gastos en DM, aunque a expensas de EE.UU. y Canadá. Los gastos promedio por paciente en el resto de los países de la región son bajos, con cifras menores de 2,000 US\$ en la mayoría de los países del Caribe 3 .

En el sur y centro de América, por su parte, la prevalencia es del $9.4 \%$, con predicciones de 
incrementarse al 11.9\% en 2040. Los gastos promedio por paciente diabético fluctúan entre 1,169 y 2,027 US\$3.

Uno de los retos fundamentales a enfrentar en relación con la situación de la DM a nivel global es, justamente, la afectación proporcionalmente superior en países de medianos y bajos ingresos (y dentro de éstos, los de $A L-C$ ), con predicciones de incrementarse en las próximas décadas, unida a la pobre infraestructura con que éstos cuentan para afrontarla. Esto hace indispensable que las estrategias implementadas sean lo suficientemente efectivas en términos costo-beneficio.

Existe consenso en que el comportamiento epidémico de la DM puede revertirse, en buena medida, con cambios en el estilo de vida; por otro lado, el diagnóstico temprano a partir de pesquisa activa y un tratamiento adecuado que garantice el buen control metabólico influyen favorablemente en evitar las complicaciones y disminuir la mortalidad. Todo esto es indicativo de que tanto el desarrollo de la enfermedad como la aparición de complicaciones y la muerte como consecuencia de ésta resultan en gran medida evitables $y$, consecuentemente, modificables con políticas y estrategias adecuadas.

Lo antes expuesto apoya la necesidad de estimados confiables de la carga de la DM para orientar y evaluar políticas encaminadas no sólo al diagnóstico temprano y el tratamiento eficaz, sino también, y de una manera prioritaria, a la prevención.

La utilización de nuevas variantes metodológicas, que complementan la información brindada por los indicadores epidemiológicos «convencionales» en relación con la magnitud de la afectación por DM, permite un análisis más integral y exhaustivo al afecto de orientar estas políticas.
La descripción consistente y comparable de la carga de las enfermedades, así como de los factores de riesgo que las causan, es esencial para la toma de decisiones y la planificación de las políticas y los recursos. La información disponible sobre mortalidad y morbilidad es fragmentada, y muchas veces inconsistente en todas las regiones del mundo. Por tanto, resulta imprescindible un marco para integrar, validar, analizar y diseminar estas informaciones al efecto de estimar la importancia comparativa de las distintas enfermedades como causa de mortalidad prematura, pérdida de salud y discapacidad en las distintas poblaciones ${ }^{5,6}$.

Éste, justamente, ha sido el propósito fundamental de los estudios de carga de enfermedad (burden of disease). El primer estudio global de carga de enfermedad (Global Burden of Disease Study [GBD]) fue referido al año 1990 (GBD 1990)7, aunque posteriormente se han realizado otros estudios globales ${ }^{8-30}$ y adicionalmente se han llevado a cabo numerosos estudios nacionales ${ }^{31-39}$.

La metodología del GBD 1990 incluyó la introducción de un nuevo indicador para estimar, de manera integrada, las pérdidas asociadas a la mortalidad y la morbilidad, los años de vida saludables perdidos (disability-adjusted lifeyears [DALY]). Ésta es una medida de tiempo que integra los años de vida perdidos por mortalidad prematura (YLL) y los años de vida perdidos por vivir en condiciones no óptimas de salud (YLD). De modo que un DALY se corresponde con un año de vida saludable perdido ${ }^{6,7}$.

Estimados de la carga de la DM en términos de DALY y sus dos componentes (YLL y YLD) han sido reportados en los diferentes estudios globales de carga $^{7-10,13,14,17,20,23,27,28-30}$.

Dada la relevancia de la DM como problema de salud en la región, integrar y analizar los resultados 
de los estudios de carga, tanto globales como nacionales, constituye un punto de partida imprescindible a los efectos de reformular y evaluar estrategias y políticas encaminadas a su prevención y control. El presente trabajo toma como base los resultados reportados por el GBD 2015 para esta enfermedad en AL-C.

\section{ANTECEDENTES DE LOS ESTUDIOS GLOBALES DE CARGA DE ENFERMEDAD}

El primer estudio global de carga (GBD 1990) estuvo encaminado a proporcionar estimaciones de la carga de 107 enfermedades o lesiones y 10 factores de riesgo para 8 regiones geográficas a nivel mundial7. Sus resultados han sido utilizados por agencias gubernamentales y no gubernamentales para informar de las prioridades en salud, el desarrollo de políticas y la asignación de recursos ${ }^{5}$.

En el año 2000, la Organización Mundial de la Salud (OMS) comenzó a publicar actualizaciones regulares del GBD para un número mayor de regiones, enfermedades y factores de riesgo, y basadas en principios metodológicos más sólidos y series de datos más extensas ${ }^{8-10}$.

Sin embargo, el estudio del año 2010 (GBD 2010) constituyó el mayor esfuerzo por generar estimados confiables y comparables de la carga de las enfermedades, lesiones y factores de riesgo desde el GBD 1990; en este caso para dos años: 1990 y 2010. En este estudio, coordinado por el Instituto de Métricas de Salud y Evaluación (Institute for Health Metrics and Evaluation), participaron 488 investigadores de 50 países y 303 instituciones. La fortaleza del GBD 2010 estuvo basada en dos aspectos fundamentales: el perfeccionamiento progresivo de la metodología para este tipo de estudio y la disponibilidad de nuevas fuentes de datos primarios (encuestas demográficas y encuestas mundiales y nacionales de salud). El GBD 2010 incluyó 291 enfermedades y lesiones, 1,160 secuelas de éstas y 67 factores de riesgo para 187 países y 21 regiones del mundo. La descripción detallada de su metodología, fuentes de obtención de datos y resultados ha sido objeto de múltiples publicaciones ${ }^{11-19}$.

Una recomendación del GBD 2010 fue llevar a cabo actualizaciones sistemáticas; y sobre esta base se desarrolló un estudio posterior referido al año 2013 (GBD 2013), el cual incluyó 306 enfermedades y 2,337 secuelas para 188 países. Algunas limitaciones identificadas en el GBD 2010, relativas a aspectos metodológicos y fuentes de datos específicos, fueron superadas en el GBD 2013. Descripciones detalladas de su metodología y resultados pueden ser consultadas en diferentes publicaciones ${ }^{20-27}$.

Con la intención de aportar información cada vez más completa y fiable se desarrolló el GBD 2015, que incluyó 310 enfermedades y aportó resultados a nivel global, regional (21 regiones), nacional (195 países) y territorial (subnacional para 8 países) ${ }^{28-30}$.

\section{LA DIABETES MELLITUS EN EL MARCO DE LOS ESTUDIOS DE CARGA DE ENFERMEDAD}

Los DALY ofrecen un peso equivalente a las enfermedades letales y a aquéllas que no conducen a la muerte pero que afectan a la capacidad de los individuos de manera temporal o permanente, con la consecuente carga sobre los servicios de salud y los costos que se derivan de una atención continua y muchas veces especializada. La OMS recomienda la utilización de este indicador para evaluar los beneficios en salud en los estudios de costo-efectividad, 
debido a que integrar las pérdidas por morbilidad y por mortalidad favorece la comparación entre diferentes alternativas de intervención ${ }^{40,41}$.

La DM genera una importante carga tanto en términos de mortalidad como por la discapacidad asociada a sus complicaciones. Los DALY permiten cuantificar el impacto (sobre los resultados fatales y no fatales) de las intervenciones y políticas en salud encaminadas a disminuir su afectación. Adicionalmente, facilitan la comparación de la carga de la DM, de manera integral, entre países y regiones, lo que resulta orientativo al efecto de planificar acciones y recursos ${ }^{42,43}$.

Los estudios de carga han identificado que esta enfermedad ascendió del lugar 18 al 15 como causa de mortalidad prematura a nivel global del año 2005 al 2015, y aunque la tasa estandarizada por edad descendió en el 2.1\%, la mediana del porcentaje de cambio de la tasa bruta se incrementó en un $10.9 \%$ en el propio periodo ${ }^{28}$. Adicionalmente, la tasa de YLD estandarizada por edad se incrementó en un 5.4\% de 2005 a 2015; en este último año la DM ocupó el sexto lugar por pérdidas debidas a discapacidad entre todas las causas a nivel global ${ }^{29}$, mientras que la de DALY tuvo un ascenso del 1.6\% en el mismo periodo, pasando de la posición 16 a la 11 como generadora de AVISA ${ }^{30}$.

Paralelamente, se han desarrollado investigaciones que utilizan estas métricas para cuantificar, específicamente, la afectación por DM a nivel regional y nacional42-49.

En consistencia con los estudios globales, los realizados a nivel regional y nacional identifican a la DM entre las enfermedades generadoras de mayor carga en términos de AVISA, y coinciden en insistir en la pertinencia de estimar la magnitud de su afectación a partir de estos indicadores, al efecto de orientar las políticas encaminadas a su prevención y control $42-49$.

La DM constituye la cuarta causa de años de vida saludable perdidos en AL-C, llegando a ocupar el primer lugar en varios países de la región: México, Antigua y Barbuda, Barbados, Dominica, Granada, Jamaica, Puerto Rico, Santa Lucía, San Vicente y las Granadinas, y Trinidad y Tobago ${ }^{30}$.

\section{Algunos resultados del Global Burden of Disease Study 2015 para la diabetes mellitus}

Los resultados presentados (integrados y resumidos en tablas y figuras para este trabajo) provienen del GBD 2015, coordinado por el Instituto de Métricas de Salud y Evaluación, y están disponibles en el sitio web: http://ghdx.healthdata.org/gbd-results-tool.

La metodología para el cálculo de los YLL, YLD y DALY en el GBD 2015 puede consultarse en las correspondientes publicaciones ${ }^{28-30}$.

Los aportes porcentuales de los YLL e YLD al total de DALY fueron calculados para este trabajo a partir de las tasas de YLL, YLD y DALY reportadas por el GBD 2015.

El listado de regiones (21), países (195) y subnacional (para 8 países) del GBD 2015 puede encontrarse en http://www.healthdata.org/sites/default/files/files/ Projects/GBD/GBDRegions_countries.pdf.

Para AL-C, la distribución de los países según regiones es la siguiente:

- Sur de Latinoamérica: Argentina, Chile y Uruguay.

- Latinoamérica tropical: Brasil y Paraguay. 
- Latinoamérica andina: Bolivia, Ecuador y Perú.

- Caribe: Antigua y Barbuda, Bahamas, Barbados, Belice, Bermudas, Cuba, Dominica, República Dominicana, Granada, Guyana, Haití, Jamaica, Puerto Rico, Santa Lucía, San Vicente y las Granadinas, Surinam, Trinidad y Tobago e Islas Vírgenes.

- Latinoamérica central: Colombia, Costa Rica, El Salvador, Guatemala, Honduras, México, Nicaragua, Panamá y Venezuela.

La tabla 1 muestra las tasas de YLL, YLD y DALY, con sus respectivos intervalos de confianza del $95 \%$ (IC 95\%), para las 21 regiones. Tres regiones de AL-C exceden los valores globales para los tres indicadores (Caribe, Latinoamérica central y Latinoamérica tropical). El Caribe muestra la mayor tasa de YLL entre las regiones de AL-C (757.01/100,000) y Latinoamérica central las mayores tasas de YLD y AVISA (639.08 y 1,330.53/100,000, respectivamente). Otras regiones que destacan por sus tasas de AVISA superiores al valor global son: Oceanía, sur de África subsahariana, sudeste de Asia, países de altos ingresos de Norteamérica, norte de África y Medio Oriente y Europa central.

Un aspecto importante en el análisis de los DALY es determinar el aporte a éstos de cada uno de sus componentes (YLL y YLD). En el caso de la DM, un mayor aporte de la mortalidad puede relacionarse con insuficiencias en la atención a las personas que viven con la enfermedad. En la figura 1 se muestra el aporte a los DALY por DM de cada componente para las 21 regiones.

Con la excepción de la Latinoamérica andina, el resto de las regiones de AL-C supera el valor global de aporte de la mortalidad (48\%). Destacan como las regiones con mayores porcentajes de YLL el Caribe (58\%) Latinoamérica tropical (55\%).
Del resto de las regiones, Oceanía, las cuatro de África subsahariana y el sur y sudeste de Asia muestran aportes de mortalidad superiores a los valores globales, mientras que las tres regiones de Europa, Australia, los países de altos ingresos de Norteamérica y Asia-Pacífico, este y centro de Asia tienen aportes de mortalidad por debajo de la media global.

La figura 2 muestra el aporte de la DM al total de YLL, YLD y DALY para las regiones de AL-C. Los valores de este indicador son una medida de la carga relativa de la DM en comparación con el resto de las enfermedades. Se evidencia que en las cinco regiones la DM hace un mayor aporte a la mortalidad prematura (YLL) y a las AVISA que el correspondiente valor a nivel global $(1.8$ y $2.6 \%$, respectivamente). Destacan por el aporte de la DM a los YLL totales, Latinoamérica central (4.9\%) y el Caribe (3.7\%). Las propias regiones muestran el mayor aporte de la DM a los AVISA totales (5.6 y $4.1 \%$, respectivamente). Con respecto a los YLD, sólo en Latinoamérica central (6.8\%) y el Caribe (4.9\%) la DM hace un aporte mayor que a nivel global (4.2\%).

En la tabla 2 se muestran las tasas de AVISA (con el correspondiente IC 95\%), así como los porcentajes del total de AVISA correspondientes a los YLL e YLD por países de AL-C. Se evidencia que 27 de los 35 países (un 77\%) tiene un aporte de la mortalidad que supera el correspondiente al valor global (48\%) (Fig. 1). Los 8 países con aporte de la mortalidad menor al valor mundial son: Costa Rica (29.7\%), Cuba (35.4\%), Colombia (37.7\%), Islas Vírgenes (42.2\%), Chile (43.4\%), Panamá (44.4\%), Bolivia (44.6\%) y Perú (44.8\%). Los mayores aportes de los YLL (más del 60\%) corresponden a países del Caribe (Belice, Granada, Guyana, Haití, Jamaica, Santa Lucía, San Vicente y las Granadinas, y Trinidad y Tobago).

En relación con las tasas de AVISA, 24 de los 35 países (69\%) exceden el correspondiente valor 
TABLA 1. Tasas de YLL, YLD y DALY como consecuencia de la DM por regiones. Resultados del GBD 2015

\begin{tabular}{|c|c|c|c|}
\hline Regiones & YLL (IC 95\%)* & YLD (IC 95\%)* & DALY (IC 95\%)* \\
\hline Global & $417.49(402.87-435.52)$ & $452.59(312.62-617.70)$ & $870.08(725.67-1,032.59)$ \\
\hline 1. Asia central & $315.40(298.72-334.47)$ & $403.92(276.31-554.13)$ & $719.33(590.75-870.45)$ \\
\hline 2. Europa central & $313.02(302.76-323.83)$ & $600.02(410.32-818.71)$ & $913.04(720.23-1,133.54)$ \\
\hline 3. Europa del cste & $156.60(146.73-167.24)$ & $449.89(307.32-615.92)$ & $606.49(459.09-770.36)$ \\
\hline 4. Australia & $231.29(213.68-249.30)$ & $466.76(321.82-638.92)$ & $698.05(552.65-871.79)$ \\
\hline 5. Altos ingresos en Asia-Pacífico & $202.35(190.29-214.35)$ & $481.50(330.97-664.02)$ & $683.85(530.16-862.45)$ \\
\hline 6. Altos ingresos Norteamérica & $365.39(350.26-381.16)$ & $818.11(568.06-1,115.59)$ & $1183.50(934.18-1,479.59)$ \\
\hline 7. Sur de Latinoamérica & $374.26(351.56-396.50)$ & $372.48(255.62-510.91)$ & $746.74(622.84-887.64)$ \\
\hline 8. Europa occidental & $250.19(239.49-260.53)$ & $435.01(301.82-593.74)$ & $685.20(550.11-845.25)$ \\
\hline 9. Latinoamérica andina & $324.33(299.06-352.06)$ & $360.92(245.53-493.22)$ & $685.25(562.64-822.80)$ \\
\hline 10. Caribe & $757.01(699.04-824.41)$ & $537.41(371.25-733.03)$ & $1294.43(1,109.67-1,496.06)$ \\
\hline 11. Latinoamérica central & $691.45(671.92-711.63)$ & $639.08(439.44-873.98)$ & $1330.53(1,130.26-1,572.74)$ \\
\hline 12. Latinoamérica tropical & $557.97(530.31-585.50)$ & $460.71(316.60-631.84)$ & $1,018.68(868.42-1,192.83)$ \\
\hline 13. Norte de África y Medio Oriente & $418.87(390.63-448.69)$ & $660.14(448.38-917.01)$ & $1,079.01(866.71-1,335.19)$ \\
\hline 14. Sur de Asia & $573.15(530.92-617.26)$ & $371.14(254.17-508.68)$ & $944.29(813.76-1,088.75)$ \\
\hline 15. África subsahariana central & $366.08(219.95-630.14)$ & $259.93(179.34-351.40)$ & $626.00(446.67-901.22)$ \\
\hline 16. África subsahariana del este & $295.90(239.47-363.76)$ & $196.04(133.62-265.75)$ & $491.94(405.44-588.41)$ \\
\hline 17. África subsahariana del sur & $1,162.75(1,028.27-1,327.76)$ & $584.88(399.74-800.26)$ & $1,747.63(1,511.81-1,997.89)$ \\
\hline 18. África subsahariana del oeste & $265.96(217.81-341.52)$ & $150.94(102.94-210.00)$ & $416.90(343.36-508.93)$ \\
\hline 19. Este de Asia & $208.96(196.42-222.62)$ & $433.89(294.93-603.32)$ & $642.85(501.80-811.46)$ \\
\hline 20. Sudeste de Asia & $694.41(618.80-783.64)$ & $585.36(396.86-806.20)$ & $1,279.77(1,073.89-1,505.85)$ \\
\hline 21. Oceanía & $1,764.05(1,317.83-2,402.13$ & $737.81(505.94-1,006.47)$ & $2,501.86(1,963 \cdot 35-3,191.09)$ \\
\hline
\end{tabular}

*Tasas por 100,000 habitantes.

Fuente: Resultados del GBD 2015. Disponible en: http://ghdx.healthdata.org/gbd-results-tool

global (870.08/100,000) (Tabla 1). Los países con cifras de este indicador inferiores al propio valor mundial son: Perú (467.93), Costa Rica (525.01), Colombia (659.88), Bolivia (674.85), Chile (704.49), Honduras (709.7), Uruguay (729.21), República Dominicana (761.44), Argentina (765.56), Nicaragua (830.82) y Cuba (852.31).

También a partir de este indicador, los valores más desfavorables (mayores tasas de AVISA) corresponden a países del Caribe, a los que se añade México.

\section{ALGUNOS COMENTARIOS SOBRE EL PANORAMA DE AMÉRICA LATINA Y EL CARIBE EN RELACIÓN CON LA DIABETES MELLITUS}

Según Chen, et al., las causas del comportamiento epidémico que ha mostrado la DM de tipo 2 (DM2), que representa alrededor del 90\% de los casos de DM, en las últimas décadas a nivel global involucra un complejo grupo de sistemas genéticos y epigenéticos, interactuando dentro de una 


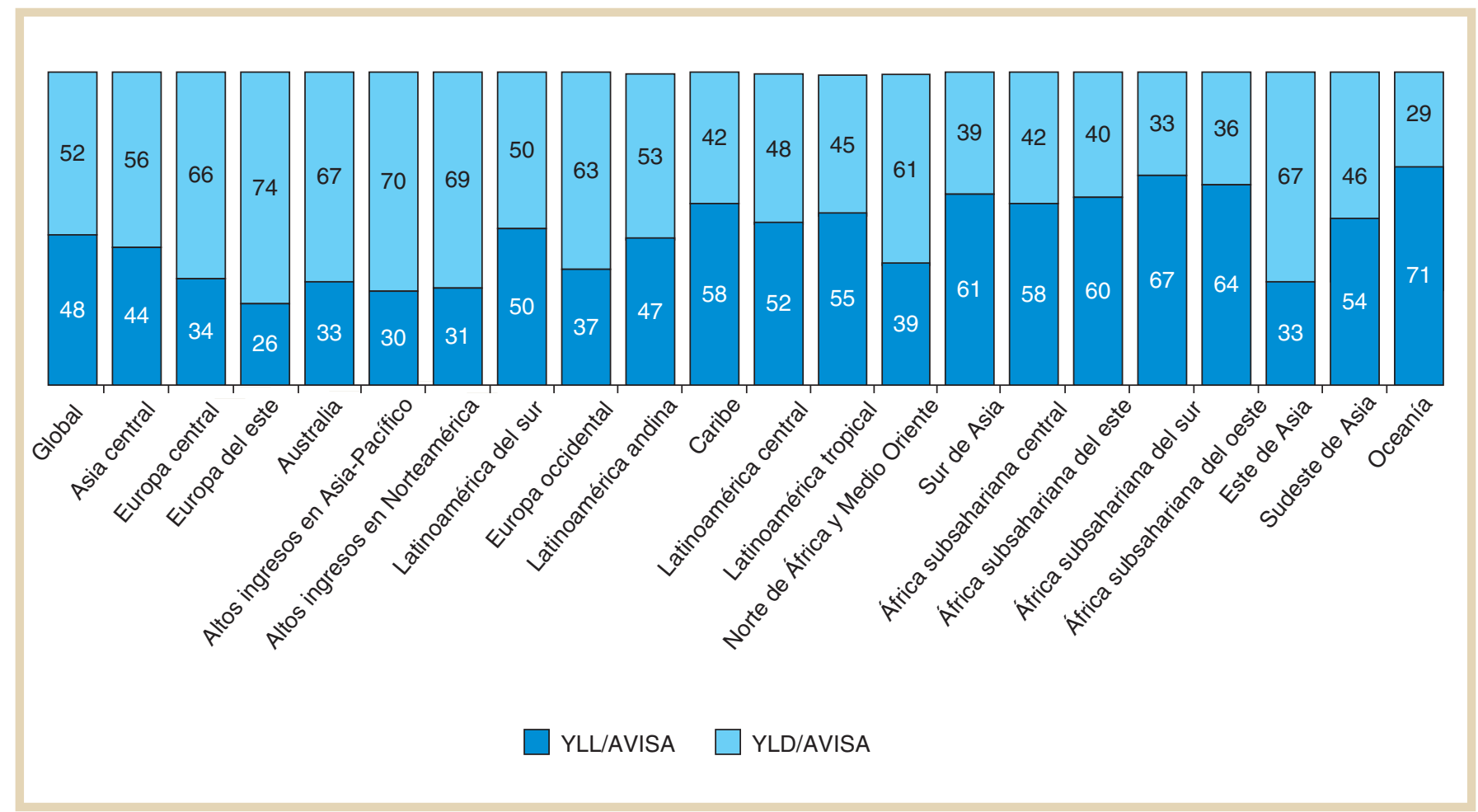

Fıgura 1. Porcentajes del total de DALY por DM correspondientes a YLL e YLD según regiones. Resultados del GBD 2015. Los porcentajes fueron calculados a partir de las tasas de YLL, YLD y DALY reportadas por el GBD 2015. (Disponible en: http://ghdx.healthdata.org/gbd-results-tool).

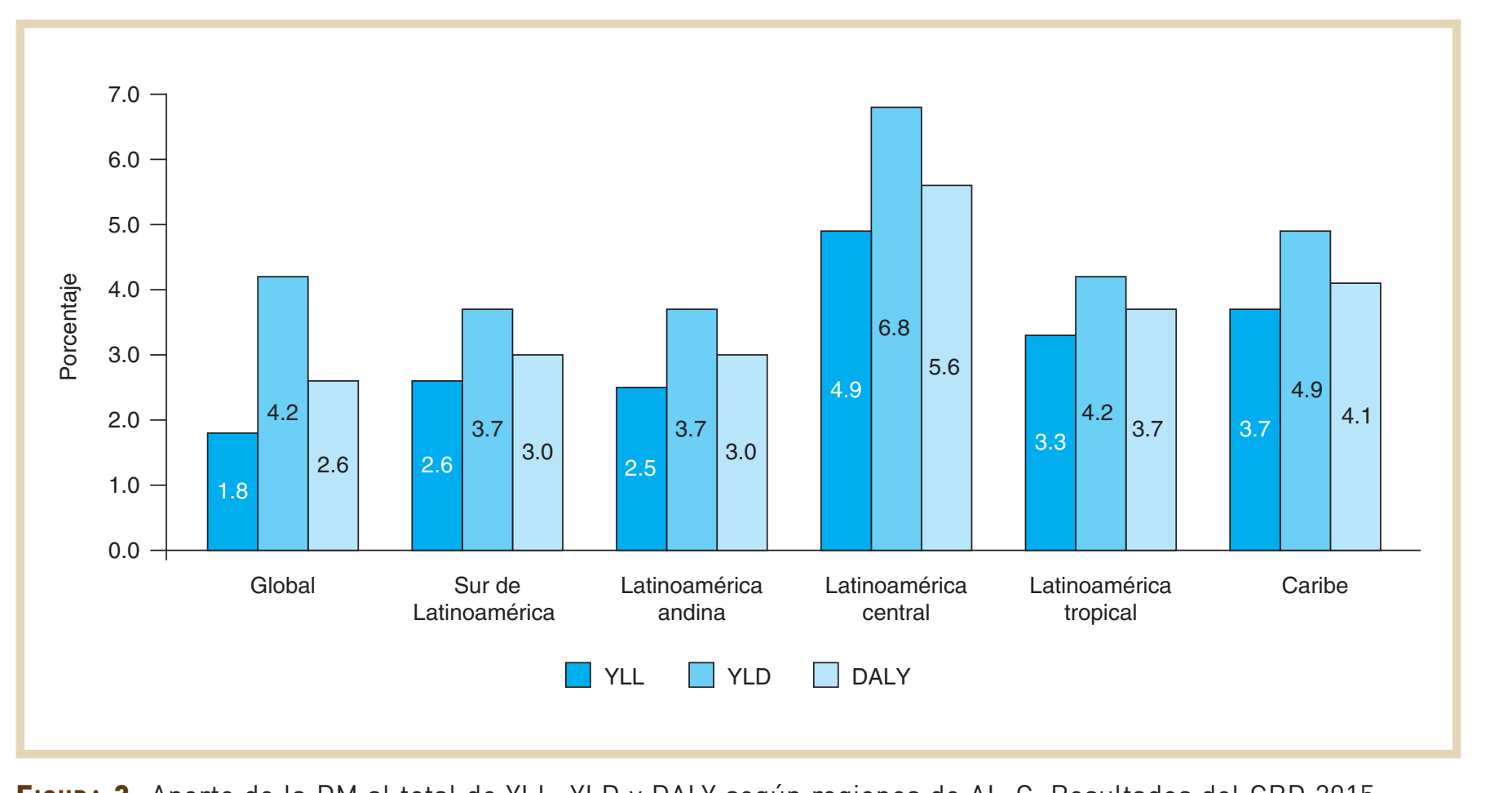

Figura 2. Aporte de la DM al total de YLL, YLD y DALY según regiones de AL-C. Resultados del GBD 2015. (Disponible en: http://ghdx.healthdata.org/gbd-results-tool). 
TABLA 2. Tasa de DALY por DM y aporte de la mortalidad y la morbilidad al total de DALY según países de AL-C

\begin{tabular}{|c|c|c|c|c|c|c|}
\hline \multirow{2}{*}{$\begin{array}{l}\text { Región } \\
\text { Sur de Latinoamérica }\end{array}$} & \multirow{2}{*}{$\begin{array}{l}\text { País } \\
\text { Argentina }\end{array}$} & \multirow{2}{*}{$\begin{array}{c}\text { Tasa de DALY* } \\
765.56\end{array}$} & \multicolumn{2}{|c|}{ Tasa de DALY (IC 95\%) } & \multirow{2}{*}{$\begin{array}{c}\text { Mortalidad (\%) } \\
52.5\end{array}$} & \multirow{2}{*}{$\begin{array}{c}\text { Morbilidad (\%) } \\
47.5\end{array}$} \\
\hline & & & 644.65 & 904.61 & & \\
\hline & Chile & 704.49 & 567.72 & 865.28 & 43.4 & 56.6 \\
\hline & Uruguay & 729.21 & 611.91 & 859.40 & 52.8 & 47.2 \\
\hline \multirow{2}{*}{$\begin{array}{l}\text { Latinoamérica } \\
\text { tropical }\end{array}$} & Brasil & $1,015.29$ & 865.29 & $1,191.44$ & 54.6 & 45.4 \\
\hline & Paraguay & $1,124.67$ & 952.68 & $1,322.23$ & 59.5 & 40.5 \\
\hline \multirow[t]{3}{*}{ Latinoamérica andina } & Bolivia & 674.85 & 539.92 & 817.42 & 44.6 & 55.4 \\
\hline & Ecuador & $1,114 \cdot 53$ & 915.59 & $1,342.15$ & 50.5 & 49.5 \\
\hline & Perú & 467.93 & 379.65 & 566.49 & 44.8 & 55.2 \\
\hline \multirow[t]{18}{*}{ Caribe } & Antigua y Barbuda & $1,775.17$ & $1,503 \cdot 37$ & $2,083.78$ & 58.9 & 41.1 \\
\hline & Bahamas & $1,529.95$ & $1,284.09$ & $1,804.79$ & 53.4 & 46.6 \\
\hline & Barbados & $2,704.77$ & $2,312.19$ & $3,175.08$ & 58.3 & 41.7 \\
\hline & Belice & $1,324.85$ & $1,145.56$ & $1,530.80$ & 65.1 & 34.9 \\
\hline & Bermudas & $1,081.16$ & 903.79 & $1,282.97$ & 50.9 & 49.1 \\
\hline & Cuba & 852.31 & 669.89 & $1,056.81$ & 35.4 & 64.6 \\
\hline & Dominica & $2,202.61$ & $1,851.88$ & $2,602.68$ & 56.0 & 44.0 \\
\hline & República Dominicana & 761.44 & 650.51 & 890.91 & 56.0 & 44.0 \\
\hline & Granada & $1,995.58$ & $1,742.95$ & $2,282.94$ & 66.0 & 34.0 \\
\hline & Guyana & $2,385.82$ & $2,079.96$ & $2,753.42$ & 69.2 & 30.8 \\
\hline & Haití & $1,159 \cdot 92$ & 898.32 & $1,456.91$ & 66.7 & 33.3 \\
\hline & Jamaica & $2,164.67$ & $1,868.95$ & $2,489.92$ & 67.2 & 32.8 \\
\hline & Puerto Rico & $2,204.63$ & $1,892.77$ & $2,550.51$ & 58.6 & 41.4 \\
\hline & Santa Lucía & $1,988.76$ & $1,739.73$ & $2,267.02$ & 64.8 & 35.2 \\
\hline & San Vicente y las Granadinas & $2,638.62$ & $2,336.12$ & $2,993.77$ & 68.7 & 31.3 \\
\hline & Surinam & $1,454.34$ & $1,234.08$ & $1,710.15$ & 58.8 & 41.2 \\
\hline & Trinidad y Tobago & $3,970.93$ & $3,479.53$ & $4,478.90$ & 69.3 & 30.7 \\
\hline & Islas Vírgenes & $2,278.94$ & $1,932.18$ & $2,665 \cdot 34$ & 42.2 & 57.8 \\
\hline \multirow[t]{9}{*}{ Latinoamérica central } & Colombia & 659.88 & 528.19 & 814.59 & 37.7 & 62.3 \\
\hline & Costa Rica & 525.01 & 406.25 & 662.91 & 29.7 & 70.3 \\
\hline & El Salvador & $1,175.78$ & 978.70 & $1,398.50$ & $51, .7$ & 48.3 \\
\hline & Guatemala & $1,275.68$ & $1,058.63$ & $1,510.92$ & 53.5 & 46.5 \\
\hline & Honduras & 709.70 & 558.69 & 858.81 & 48.8 & 51.2 \\
\hline & México & $1,776.32$ & $1,524.89$ & $2,100.32$ & $54 \cdot 3$ & 45.7 \\
\hline & Nicaragua & 830.82 & 697.66 & 975.67 & 55.0 & 45.0 \\
\hline & Panamá & $1,038.06$ & 839.54 & $1,267.01$ & 44.4 & 55.6 \\
\hline & Venezuela & $1,030.65$ & 849.95 & $1,230.99$ & 51.5 & 48.5 \\
\hline
\end{tabular}

*Tasa por 100,000 habitantes.

Los porcentajes fueron calculados a partir de las tasas de YLL, YLD y DALY reportadas por el GBD 2015.

Fuente: Resultados del GBD 2015. Disponible en: http://ghdx.healthdata.org/gbd-results-tool 
igualmente compleja estructura social que determina tanto el comportamiento como la influencia del ambiente ${ }^{2}$.

Los factores genéticos han sido considerados una explicación para las diferencias étnicas en el riesgo de desarrollar DM y sus complicaciones. Estudios genéticos han identificado más de 50 locus significativamente asociados con la DM, y al menos 14 de ellos se han replicado en poblaciones hispanas ${ }^{50,51}$.

Diversas investigaciones han reportado una asociación positiva de la ascendencia genética de nativos americanos con el índice de masa corporal y el riesgo de $\mathrm{DM}_{2}{ }^{52-55}$. Sin embargo, estudios recientes sugieren que esta relación es mucho más compleja que lo que en un inicio se consideró56. Por ejemplo, se ha observado que después de controlar por el estatus socioeconómico, la asociación entre la ascendencia genética y el riesgo de $\mathrm{DM}_{2}$ se debilita54.

De modo que la evidencia más actual indica que la DM2 es un trastorno poligenético que se desarrolla debido a la compleja interacción entre múltiples genes y factores ambientales. El modo en que interactúan estos genes entre sí y con el medio ambiente para producir DM2 todavía es poco conocido57.

La influencia de factores ambientales en el desarrollo y el pronóstico de la DM está ampliamente documentada en la literatura médica ${ }^{2,58}$. Estos factores están estrechamente vinculados a los determinantes sociales de la salud y, a diferencia del código genético, resultan modificables ${ }^{59}$.

De modo que para analizar la situación de AL-C con respecto a la $\mathrm{DM}$, sin subestimar la influencia genética, se impone enfatizar en factores de tipo social determinantes de desigualdades en salud.
Se dispone de suficiente evidencia científica en relación con la existencia de inequidades en la prevención, diagnóstico, tratamiento, control y monitoreo de la DM. La mayoría de la información disponible sobre el tema procede de investigaciones llevadas a cabo en países desarrollados, y confirma que, aun en aquéllos de muy altos ingresos y con sistemas de salud universal, se identifican inequidades socioeconómicas y étnicas en la provisión de cuidados de salud a los pacientes diabéticos ${ }^{60-66}$.

A pesar de que en los últimos años las desigualdades en relación con los ingresos han disminuido en $A L-C$, ésta continúa siendo la región más inequitativa del mundo. Estas diferencias son también notorias en lo relativo a educación y acceso a servicios básicos como la salud ${ }^{67}$.

Un estudio encaminado a identificar las desigualdades en salud existentes entre países de AL-C (20 países), en el periodo 2005-2010, confirmó importantes brechas entre éstos y reafirmó a la región como la más inequitativa a nivel global ${ }^{68}$.

En pocos países de América Latina, como México ${ }^{69}$, Chile $^{70}$ y Colombia ${ }^{71}$, entre otros, se ha estudiado la relación de los determinantes sociales con el desarrollo y control de la DM; además, estos estudios tienen la limitante de que no han incluido la medición de las inequidades. La multidimensionalidad del concepto y la falta de accesibilidad a la información requerida han afectado al desarrollo de investigaciones encaminadas a medir inequidades en la región ${ }^{72}$.

Más numerosas son las investigaciones de este tipo llevadas a cabo en poblaciones oriundas de AL-C residentes en países desarrollados; por ejemplo, población originaria del Caribe residente en el Reino Unido $^{73}$ e hispanos residentes en EE.UU.59,74. Éstas han permitido reafirmar la influencia de las 
desigualdades socioeconómicas y étnicas en la morbimortalidad por DM, identificando mayor prevalencia de la enfermedad y sus complicaciones, mayor riesgo de morir, peor control glucémico y tasas más bajas de automonitoreo de la glucemia en las llamadas minorías étnicas. Estos resultados se asocian, esencialmente, a limitaciones en el acceso a alimentación saludable, a lugares adecuados y seguros para la realización de ejercicios físicos y a servicios de salud de calidad, entre otros factores.

Otro aspecto que influye en la situación de la DM en AL-C es la insuficiente efectividad de los sistemas de salud en la prevención y el tratamiento de las enfermedades crónicas en general y la DM en particular. Aun cuando existen pocos estudios sobre la calidad en la atención, éstos coinciden en reportar grandes diferencias entre las guías clínicas oficiales y los cuidados que en la práctica se ofrecen a los pacientes. Esto, unido al pobre enfoque a la prevención, hace de estas enfermedades un gran reto para AL-C75.

La DM impone una importante carga económica a los individuos y la sociedad en todas las regiones y países de AL-C. Se evidencia una brecha entre los gastos en salud y los costos del cuidado del paciente diabético, lo que se asocia a resultados adversos, tales como alta frecuencia de complicaciones, discapacidad y mortalidad prematura, así como falta de confianza de los pacientes en los servicios de salud $^{76}$.

El acceso limitado a cuidados de salud adecuados ocasiona que los costos indirectos (causados por las afectaciones productivas debidas a discapacidad y mortalidad prematura) excedan a los costos directos por cuidados de salud ${ }^{7}$. Se estima que el $55 \%$ de los costos de la DM en AL-C son atribuibles a mortalidad prematura y discapacidad temporal y permanente ${ }^{77}$.
Las predicciones epidemiológicas para las enfermedades crónicas no trasmisibles en la región permiten asegurar que sus costos adquirirán dimensiones catastróficas para los pacientes y los sistemas de salud, lo que ocasionará que la alta carga económica que representa la DM enfrentará una fuerte competencia con otras enfermedades en la asignación de recursos. De modo que se impone reformular paradigmas y programas para enfrentar esta enfermedad, enfocados a una redistribución de los recursos con una mayor inversión en prevención y promoción de salud, e involucrando de manera activa a los pacientes, proveedores de salud, organizaciones sanitarias y comunidad77.

\section{CONSIDERACIONES FINALES}

La DM representa una importante carga para los países de AL-C en términos de AVISA. Tres de las cinco regiones tienen tasas de YLL, YLD y DALY que exceden los valores globales, y cuatro aportes de la mortalidad superiores al propio valor para el mundo. Las cinco regiones exceden el valor global de aporte de la DM a los YLL y AVISA totales. Aproximadamente tres cuartas partes de los países de la región tiene tasas de AVISA que superan las mundiales.

Un aspecto en que se debe enfatizar es que para la mayoría de los países la contribución de la mortalidad a las AVISA excede al valor global, lo que es indicativo de insuficiencias en el tratamiento, monitoreo y control de las personas con DM en la región.

El comportamiento antes descrito no resulta homogéneo para las cinco regiones. Las que consistentemente muestran una mayor carga son el Caribe y Latinoamérica central, mientras que el sur de Latinoamérica y Latinoamérica andina tienen valores similares a los correspondientes valores globales para la mayoría de los indicadores. 
Al analizar la situación de la DM en AL-C, aun cuando no es posible desestimar la influencia genética, es imprescindible enfatizar en aspectos relativos a los determinantes sociales y a las estrategias y políticas en salud en la región, concretamente en: a) desigualdades en salud, que ponen en situación de desventaja a algunos grupos sociales en lo relativo a calidad de la alimentación, las posibilidades de llevar a cabo actividad física y el acceso a servicios de salud de calidad, entre otros; b) incumplimientos de las guías clínicas de atención a estos pacientes; c) estrategias y políticas en salud que no priorizan la prevención, y d) insuficiente asignación de recursos destinados al diagnóstico temprano, el tratamiento eficaz y el monitoreo de la DM, lo cual conduce a un inadecuado control de la enfermedad y al desarrollo temprano de complicaciones, con la correspondiente carga por discapacidad y mortalidad temprana.

Existe evidencia suficiente de que tanto el desarrollo de la DM como la aparición de complicaciones y la muerte como consecuencia de ésta resultan en gran medida evitables y, consecuentemente, modificables con políticas y estrategias adecuadas.

De modo que se impone un cambio de paradigmas para enfrentar esta enfermedad en la región, con estrategias que impliquen una redistribución de los recursos con una mayor inversión en prevención y promoción de la salud, y con un enfoque comunitario.

Paralelamente, es indispensable llevar a cabo investigaciones destinadas a medir las inequidades en salud en la región, como paso previo imprescindible para el establecimiento de intervenciones encaminadas a disminuir las brechas, actuando directamente en sus causas.

La medición consistente y sistemática de la carga de la DM, a partir de diferentes alternativas metodológicas, y el análisis integral de estas mediciones resultan esenciales para evaluar las estrategias actuales y orientar hacia nuevas propuestas.

\section{BIBLIOGRAFÍA}

1. Danaei G, Finucane M, Lu Y, Singh GM, Cowand MJ, Paciorek CJ, et al. National, regional, and global trends in fasting plasma glucose and diabetes prevalence since 1980: systematic analysis of health examination surveys and epidemiological studies with 370 country-years and 2,7 million participants. Lancet. 2011;378(9785):31-40.

2. Chen L, Magliano DJ, Zimmet PZ. The worldwide epidemiology of type 2 diabetes mellitus-present and future perspectives. Nat Rev Endocrinol. 2012;8(4):228-36.

3. International Diabetes mellitus Federation. IDF Diabetes Atlas 2015. Brussels, Belgium: International Diabetes mellitus Federation; 2016 [citado el 28 de julio de 2016]. Disponible en: www.idf.org/diabetes mellitus atlas

4. International Diabetes Federation. IDF Diabetes Atlas 2013. Brussels, Belgium: International Diabetes Federation; 2014 [citado el 28 de marzo de 2016]. Disponible en: www.idf.org/diabetes mellitus atlas

5. López AD, Mathers CD, Ezatti M, Jamison DT, Murray CJL. Measuring The Global Burden of Disease and Risk Factors. 1990-2001. En: López AD, Mathers CD, Ezatti M, Jamison DT, Murray CJL, editors. Global Burden of Disease and Risk Factors. New York: Oxford University Press and The World Bank; 2006.

6. Etches V, Frank J, Di Ruggiero E, Doug M. Measuring population health: A Review of Indicators. Annu Rev Public Health. 2006;27:29-55.

7. Murray CJL, López A. The Global Burden of Disease: a comprehensive assessment of mortality and disability from diseases, injuries and risk factors in 1990 and projected to 2020. Volume 1. Geneva, WHO; 1996.

8. World Health Organization. The world health report 2000. Health systems: improving performance. [Internet]. Geneva: World Health Organization; 2000 [citado el 23 de agosto de 2007]. Disponible en: http://www.who.int/whr/2000/en/whroo_en.pdf

9. World Health Organization. Death and DALY estimates for 2002 by cause for WHO Member States. [homepage en Internet]. Geneva: World Health Organization; 2004 [citado el 12 de enero de 2009]. Disponible en: http://www.who.int/evidence/bod

10. World Health Organization. World health report 2004: changing his tory. Geneva: World Health Organization; 2004 [citado el 1 de marzo de 2011]. Disponible en: http://www.who.int/whr/2004/en/whro4 en.pdf

11. Murray CJ, Ezzati M, Flaxman AD, Murray CJ, Ezzati M, Flaxman AD, et al. GBD 2010: design, definitions, and metrics. Lancet. 2012; 380(9859):2063-6.

12. Lim SS, Vos T, Flaxman AD, Lim SS, Vos T, Flaxman AD, et al. A comparative risk assessment of burden of disease and injury attributable to 67 risk factors and risk factor clusters in 21 regions, 1990-2010: a systematic analysis for the Global Burden of Disease Study 2010. Lancet. 2012;380(9859):2224-60.

13. Lozano R, Naghavi M, Foreman K, Lozano R, Naghavi M, Foreman K, et al. Global and regional mortality from 235 causes of death for 20 age groups in 1990 and 2010: a systematic analysis for the Global Burden of Disease Study 2010. Lancet. 2012;380(9859):2095-128.

14. Murray CJ, Vos T, Lozano R, Naghavi M, Flaxman AD, Michaud C, et al. Disability-adjusted life years (DALYs) for 291 diseases and injuries in 21 regions, 1990-2010: a systematic analysis for the Global Burden of Disease Study 2010. Lancet. 2012;380(9859):2197-223. 
15. Salomon JA, Vos T, Hogan DR, Gagnon M, Naghavi M, Mokdad A, et al. Common values in assessing health outcomes from disease and injury: disability weights measurement study for the Global Burden of Disease Study 2010. Lancet. 2012;380(9859):2129-43.

16. Salomon JA, Wang H, Freeman MK, Vos T, Flaxman AD, López AD, et al. Healthy life expectancy for 187 countries, 1990-2010: a systematic analysis for the Global Burden Disease Study 2010. Lancet. 2012; 380(9859):2144-62.

17. Vos T, Flaxman AD, Naghavi M, Lozano R, Michaud C, Ezzati M, et al. Years lived with disability (YLDs) for 1160 sequelae of 289 diseases and injuries 1990-2010: a systematic analysis for the Global Burden of Disease Study 2010. Lancet. 2012;380(9859):2163-96.

18. Wang H, Dwyer-Lindgren L, Lofgren KT, Lofgren KT, Rajaratnam JK, Marcus JR, et al. Age-specific and sex-specific mortality in 187 countries, 1990-2010: a systematic analysis for the Global Burden of Disease Study 2010. Lancet. 2012;380(9859):2071-94.

19. Das P, Samarasekera U. The story of GBD 2010: a "super-human" effort. Lancet. 2012;380(9859):2067-70.

20. GBD 2013 Mortality and Causes of Death Collaborators. Global, regional, and national age-sex specific all-cause and cause-specific mortality for 240 causes of death, 1990-2013: a systematic analysis for the Global Burden of Disease Study 2013. Lancet. 2015;385(9963):117-71.

21. Global Burden of Disease Pediatrics Collaboration. Global and National Burden of Diseases and Injuries Among Children and Adolescents Between 1990 and 2013 Findings from the Global Burden of Disease 2013 Study. JAMA Pediatr. 2016;170(3):267-87.

22. Wang $H$, Liddell CA, Coates MM, Mooney MD, Levitz CE, Schumacher $A E$, et al. Global, regional, and national levels of neonatal, infant, and under-5 mortality during 1990-2013: a systematic analysis for the Global Burden of Disease Study 2013. Lancet. 2014;384(9947):957-79.

23. Global Burden of Disease Study 2013 Collaborators. Global, regional, and national incidence, prevalence, and years lived with disability for 301 acute and chronic diseases and injuries for 188 countries, 19902013: a systematic analysis for the Global Burden of Disease Study 2013. Lancet. 2015;386(9995):743-800.

24. Murray CJ, Ortblad KF, Guinovart C. Global, regional, and national incidence and mortality for HIV, tuberculosis, and malaria during 1990-2013: a systematic analysis for the Global Burden of Disease Study 2013. Lancet. 2014;384(9947):1005-70.

25. Salomon JA, Haagsma JA, Davis A, de Noordhout CM, Polinder S, Havelaar AH, et al. Disability weights for the Global Burden of Disease 2013 study. Lancet Glob Health. 2015;3(11):712-23.

26. GBD 2013 Risk Factors Collaborators, Forouzanfar MH, Alexander L, Anderson HR, Bachman VF, Birykov S, Brauer M, et al. Global, regional and national comparative risk assessment of 79 behavioral, environmental and occupational, and metabolic risks or clusters of risks in 188 countries, 1990-2013: a systematic analysis for the Global Burden of Disease Study 2013. Lancet. 2015;386(10010):2287-323.

27. GBD 2013 DALYs and HALE Collaborators. Global, regional, and national disability-adjusted life years (DALYs) for 306 diseases and injuries and healthy life expectancy (HALE) for 188 countries, 1990-2013: quantifying the epidemiological transition. Lancet. 2015;386(10009): 2145-91.

28. GBD 2015 Mortality and Causes of Death Collaborators. Global, regional, and national life expectancy, all-cause mortality, and cause-specific mortality for 249 causes of death, 1980-2015: a systematic analysis for the Global Burden of Disease Study 2015. Lancet. 2016;388(10053): 1459-544.

29. GBD 2015 Disease and Injury Incidence and Prevalence Collaborators. Global, regional, and national incidence, prevalence, and years lived with disability for 310 diseases and injuries, 1990-2015: a systematic analysis for the Global Burden of Disease Study 2015. Lancet. 2016; 388(10053):1545-602.

30. GBD 2015 DALYs and HALE Collaborators. Global, regional, and national disability-adjusted life-years (DALYs) for 315 diseases and injuries and healthy life expectancy (HALE), 1990-2015: a systematic analysis for the Global Burden of Disease Study 2015. Lancet. 2016;388(10053): 1603-58.

31. Gómez Dantés H, Castro MV, Franco Marina F, Bedregal P, Rodríguez García J, Espinoza A, et al. La carga de la enfermedad en países de América Latina. Salud Pública Mex. 2011;53(Suppl 2):S72-7.

32. Ministerio de Salud de Costa Rica. Dirección Vigilancia de la Salud. Proyecto BID-MS. Estudio carga de enfermedad. Costa Rica; 2005 [citado el 7 de julio de 2016]. Disponible en: https://www.ministeriodesalud.go.cr/index.php/vigilancia-de-la-salud/analisis-de-situacion-de-salud/961-carga-enfermedad-costa-rica-ano-2005/file

33. Gènova-Maleras R, Álvarez-Martín E, Morant-Ginestar C, Fernández de Larrea-Baz N, Catalá-López F. Measuring the burden of disease and injury in Spain using disability-adjusted life years: an updated and policy-oriented overview. Public Health. 2012;126(12):1024-31.

34. Lai T, Habicht J, Kiivet RA. Measuring burden of disease in Estonia to support public health policy. Eur J Public Health. 2009;19(5):541-7.

35. Lapostolle A, Lefranc A, Gremy I, Spira A. Sensitivity analysis in summary measure of population health in France. Eur J Public Health. 2008;18(2):195-200.

36. Begg SJ, Vos T, Barker B, Stanley L, López AD. The burden of disease and injury in Australia in the new millennium: measuring health loss from diseases, injuries and risk factors. Med J Aust. 2008;188(1) 36-40.

37. Carga de Enfermedad Colombia 2005: Resultados Alcanzados. Documento Técnico Ass/1502-08. Bogotá: Pontifica Universidad Javeriana; 2008 [citado el 7 de julio de 2016]. Disponible en: http://www.javer ana.edu.co/cendex/GPES/informes/PresentacionCarga_Informe.pdf

38. Ministerio de Salud de Chile. Estudio de carga de enfermedad y carga atribuible, Chile; 2007 [citado el 7 de julio de 2016]. Disponible en: http://www.udla.cl/portales/tpgeooaf339c16/uploadImg/File/ Universidad/UDLA-Proyeccion-Carga-Enfermedad.pdf

39. Lozano R, Gómez Dantés H, Garrido Latorre F, Jiménez Corona A, Campuzano Rincón JC, Franco-Marina F, et al. La carga de enfermedad, lesiones, factores de riesgo y los desafíos para el sistema de salud en México. Salud Publica Mex. 2013;55(6):580-94.

40. Tan-Torres Edejer T, Baltussen R, Adam T, Hutubessy R, Acharya A Evans D B, et al. Making choices in health: WHO guide to cost-effectiveness analysis. Geneva, World Health Organization, 2003.

41. Mont D. Measuring health and disability. Lancet. 2007;369(9573): 1658-63.

42. Khajedaluee M, Dadgarmoghaddam M, Saeedi R, Izadi-Mood Z, Abrishami M. The Burden of Diabetes in a Developing Country. Open Journal of Preventive Medicine. 2014;4:175-81 [citado el 29 de junio de 2016]. Disponible en: http://file.scirp.org/pdf/OJPM_2014041114 001766.pdf

43. Darbà J, Kaskens L, Detournay B, Kern W, Nicolucci A, Orozco-Beltrán $D$, et al. Disability-adjusted life years lost due to diabetes in France, Italy, Germany, Spain, and the United Kingdom: a burden of illness study. Clinicoecon Outcomes Res. 2015;7:163-71.

44. Ferreira de Oliveira A, Gonçalves J, Leite I, Mendes de Andrade J, Renteria de Azevedo AS, Jourdan AM. Global burden of disease attributable to diabetes mellitus in Brazil. Cad Saúde Pública. 2009; 25(6):1234-44.

45. Escobedo J, Rodríguez-Abrego G, Aranda J, Zurita B, Ramírez T, Herrera J. Disability-adjusted life-years (DALYs) for diabetes in Mexico in 2005: a cross-sectional burden of disease analysis. Lancet. 2013;381:S46 [citado el 29 de junio de 2016]. Disponible en: http:// www.thelancet.com/journals/lancet/issue/vol381nonull/PIIS0140$6736 \% 2813 \% 29 \times 6030-1$

46. Bhutani J, Bhutani S. Worldwide burden of diabetes. Indian J Endocrinol Metab. 2014;18(6):868-70.

47. Domínguez Alonso E, Seuc Jo AH, Díaz Díaz O, Aldana Padilla D. La carga de la diabetes en Cuba, período 1990-2005. Rev Cubana Endo- 
crinol [Internet]. 2008;19(2) [citado el 20 de julio de 2012]. Disponible en: http://scielo.sld.cu/scielo.php?script=sci_arttext\&pid=S1561532008000200004 \&lng=es

48. Domínguez E, Seuc AH. Años de vida saludable perdidos debido a la diabetes en mujeres cubanas en edad fértil. Período 1990-2010. Rev ALAD. 2015;5(3):133-42.

49. Domínguez E. Disability-adjusted Life Years for Diabetes in Cuban Children and Adolescents, 1990-2010. MEDICC Review. 2016;18(4): 14-9.

50. Below JE, Gamazon ER, Morrison JV, Konkashbaev A, Pluzhnikov A, McKeigue PM, et al. Genome wide association and meta-analysis in populations from Starr County, Texas, and Mexico City identify type 2 diabetes susceptibility loci and enrichment for expression quantitative trait loci in top signals. Diabetologia. 2011;54(8):2047-55.

51. Gamboa-Meléndez MA, Huerta-Chagoya A, Moreno-Macías H, Vázquez-Cárdenas P, Ordóñez-Sánchez ML, Rodríguez-Guillén R, et al. Contribution of common genetic variation to the risk of type 2 diabetes in the Mexican Mestizo population. Diabetes. 2012;61(12):3314-21.

52. Klimentidis YC, Miller GF, Shriver MD. The relationship between European genetic admixture and body composition among Hispanics and Native Americans. American journal of human biology: the official journal of the Human Biology Council. 2009;21(3):377-82.

53. Norden-Krichmar TM, Gizer IR, Libiger O, Wilhelmsen KC, Ehlers CL, Schork NJ. Correlation analysis of genetic admixture and social identification with body mass index in a Native American community. American journal of human biology: the official journal of the Human Biology Council. 2014;26(3):347-60.

54. Flórez JC, Price AL, Campbell D, Riba L, Parra MV, Yu F, et al. Strong association of socioeconomic status with genetic ancestry in Latinos: implications for admixture studies of type 2 diabetes. Diabetologia. 2009;52(8):1528-36.

55. McCarthy MI. Genomics, type 2 diabetes, and obesity. N Engl J Med. 2010;363(24):2339-50.

56. Hu H, Huff CD, Yamamura $Y$, Wu X, Strom SS. The Relationship between Native American Ancestry, Body Mass Index and Diabetes Risk among Mexican-Americans. PLoS One. 2015;10(10):e0141260.

57. Ali O. Genetics of type 2 diabetes mellitus. World J Diabetes. 2013; 4(4):114-23.

58. Kharroubi AT, Darwish HM. Diabetes mellitus: The epidemic of the century. World J Diabetes. 2015;6(6):850-67.

59. Spanakis EK, Golden SH. Race/Ethnic Difference in Diabetes and Diabetic Complications. Curr Diab Rep. 2013;13(6) [citado el 2 de agosto de 2017]. Disponible en: https://www.ncbi.nlm.nih.gov/pmc/articles/ PMC3830901/pdf/nihms-524798.pdf\#page=4\&zoom=auto,0,493

60. Ricci-Cabello I, Ruiz-Pérez I, Olry de Labry-Lima A, Márquez-Calderón S. Do social inequalities exist in terms of the prevention, diagnosis, treatment, control and monitoring of diabetes?. A systematic review. Health and Social Care in the Community. 2010;18(6):572-87.

61. Domínguez Alonso E. Desigualdades sociales y diabetes mellitus. Rev Cub de Endocrinol. 2013;24(2):200-13.

62. Grintsova O, Maier W, Mielck A. Inequalities in health care among patients with type 2 diabetes by individual socio-economic status (SES) and regional deprivation: a systematic literature review. Inter- national Journal for Equity in Health. 2014;13:43 [citado el 2 de agosto de 2017]. Disponible en: http://www.equityhealthj.com/content/ $13 / 1 / 43$

63. Maier W, Holle R, Hunger M, Peters A, Meisinger C, Greiser KH, et al. The impact of regional deprivation and individual socio-economic status on the prevalence of Type 2 diabetes in Germany. A pooled analysis of five population-based studies. Diabet Med. 2013;30(3): e78-86.

64. Espelt A, Arriola L, Borrell C, Larranaga I, Sandin M, Escolar-Pujolar A. Socioeconomic position and type 2 diabetes mellitus in Europe 19992009: a panorama of inequalities. Curr Diabetes Rev. 2011;7(3):148-58.

65. Hsu CC, Lee CH, Wahlqvist ML, Huang HL, Chang HY, Chen L. Poverty increases type 2 diabetes incidence and inequality of care despite universal health coverage. Diabetes Care. 2012;35(11):2286-92.

66. James GD, Baker P, Badrick E, Mathur R, Hull S, Robson J. Ethnic and social disparity in glycaemic control in type 2 diabetes; cohort study in general practice 2004-9. J R Soc Med. 2012;105(7):300-8.

67. Tsounta E, Osueke Al. What is Behind Latin America's Declining Income Inequality? IMF Working Paper (WP/14/124) International Monetary Fund; 2014 [citado el 21 de agosto de /2017]. Disponible en: https://www.imf.org/external/pubs/ft/wp/2014/wp14124.pdf

68. Cardona D, Acosta LD, Bertone CL. Inequidades en salud entre países de Latinoamérica y el Caribe (2005-2010). Gac Sanit. 2013;27(4):292-7.

69. Moreno-Altamirano L, García-García JJ, Soto-Estrada G, Capraro S, Limón-Cruz D. Epidemiología y determinantes sociales asociados a la obesidad y la diabetes tipo 2 en México. Rev Med Hosp Gen Mex. 2014;77(3):114-23.

70. Martín Plaza G. Determinantes Sociales de la Salud en Chile: ¿En qué influyen? Medicina Familiar; UC [citado el 12 de agosto de 2017]. Disponible en: http://medicinafamiliar.uc.cl/html/articulos/337.html

71. Vargas-Uricoechea H, Casas-Figueroa LA. Epidemiología de la diabetes mellitus en Sudamérica: la experiencia de Colombia. Clin Invest Arterioscl. 2016;28:245-56.

72. Almeida-Filho N, Kawachi I, PellegriniFilho A, Dachs JNW. Research on Health Inequalities in Latin America and the Caribbean: Bibliometric Analysis (1971-2000) and Descriptive Content Analysis (1971-1995). Am J Public Health. 2003;93(12):2037-43.

73. Bennett NR, Francis DK, Ferguson TS, Hennis AJM, Wilks RJ, Harris EN, et al. Disparities in diabetes mellitus among Caribbean populations: a scoping review. Int J Equity Health. 2015;14:23.

74. Golden SH, Brown A, Cauley JA, Chin MH, Gary-Webb TL, Kim C, et al. Health disparities in endocrine disorders: biological, clinical, and nonclinical factors--an Endocrine Society scientific statement. J Clin Endocrinol Metab. 2012;97(9):1579-639.

75. Glassman A, Gaziano TA, BouillonBuendia CP, Guanais de Aguiar FC. Confronting the Chronic Disease Burden in Latin America and the Caribbean. Health Aff (Millwood). 2010;29(12):2142-8.

76. Barceló A, Aedo C, Rajpathak S, Robles S. The cost of diabetes in Latin America and the Caribbean. Bulletin of the World Health Organization. 2003;81(1):19-27.

77. Arredondo A. Changing paradigms and challenges: evidence on the epidemiological and economic burden of diabetes in Latin America. Diabet Med. 2017;34(7):1009-10. 